können, ebenso kann es za haltbaren Ergebnissen führen, wenn Erfahrungen über die gegenwärtigen meteorologischen Vorgänge auf die unmittelbar vorhergehende Zeit mit ihrer wenig abweichenden geographischen Gestaltung angewendet werden, wie das von EckHardt (21) umfassender, von Phlltpp (51), KNörzer (66) und GETsitz (67) für Einzelheiten geschehen ist.

Die Frage, ob man so zu ,ausreichenden" Erklärungen kommt, würde am besten vorläufig ans der Debatte ansscheiden, bis feststeht, zu welcher Erklärung man kommt, und für was sie ,ausreichen“ muss. Deshalb kann auch die in lebhafter Polemik umstrittene "Kohlensäuretheorie" von AkRHENiUs-Frech nur kurze Erwähnung finden $(5,6,17,18,68-70)$. Sie schreibt den meteorologischgeographischen Faktoren wichtigen Einfluss zu, obne ihn zu spezialisieren, hält ihn aber nicht für ausreichend, ignoriert ihn deshalb de facto und nimmt an, dass durch vulkanische Exhalation in Perioden starker Eruptivität der Kohlensäuregehalt der Laft vermehrt werde. Dadurch soll in diesen Zeiten die Ausstrahlung der irdischen Wärme in den Weltenraum verhindert werden und die Debatte dreht sich nun erstens um die Frage, ob die Theorie physikalisch haltbar sei, zweitens darum, ob die Zeiten starker vulkanischer Tätigkeit mit den geologischen Epochen warmen Klimas zusammenfallen und umgekehrt Eiszeiten mit Perioden des Nachlassens und der Seltenheit von Eruptionen.

Im ganzen and im einzelnen ist es also ein höchst nnerquicklicher, verwirrter und ungeklärter Zustand, in dem sich heute unsere Anschaunngen über das Klima der geologischen Vorzeit befinden. Es ist mehr als nur verständlich, wenn sich auf sehr vielen Seiten keine Geneigtheit zeigt, diesem Thema näher zu treten und ihm im Lehrgebäude der theoretischen Geologie mehr zu bewilligen als eine anhangsweise Berücksichtigung. Soll es jemals auf Vollberechtigung Anspruch erheben können, so werden ihm dazu, was manche erfolgreiche Ansätze gewährleisten, nur Untersuchungen zwecks Sichtung der. Kriterien oder über die klimatischen Zustände engbegrenzter Gebiete und Zeiten, sozusagen Spezialaufnahmen verhelfen, aber sicherlich nicht Zasammenfassungen, Generalisationen und die Gedankengänge erklärender Hypothesen.

\title{
Die Montagne Pelée nach der Eruption.
}

Von Bergeat (Königsberg).

LacroIx, A.: La Montagne Pelée après ses éruptions avec observations sur les éruptions du Vésuve en 79 et en 1906. Ouvrage publié par l'académie des sciences. Paris 1908.

In seinem grossen Werke "La Montagne Pelée et ses éruptions" hatte der Verf: alle Ereignisse der Eruption von Martinique und alle Veränderungen des Valkans bis in den Dezember 1904 verzeichnet und vor allem die hauptsächlichsten Erscheinungen dieser denkwürdigen Eruptionen, die heissen Wolken sowie die Bildung der Staukuppe und die damit eng zusammenhängende Emporhebung 
der gewaltigen, gegen $400 \mathrm{~m}$ hohen Felsnadel eingehend geschildert. Das vorliegende Buch bildet einesteils, gestützt auf die Beobachtungen von Lacrorx' ehemaligen Mitarbeitern Giradd, Perney und Guinoisead, eine Fortsetzung and Ergänzung jenes Hauptwerkes bis $z u$ den letzten eruptiven Äusserungen am 30. Oktober 1905, andernteils stellt es einen Vergleich an zwischen der Antilleneruption und zwei wichtigeren Eruptionen unseres Mustervulkanes, des Vesuv, insbesondere mit dem gleichfalls von Lackorx schon in früheren Publikationen geschilderten Ausbruch von 1906.

Die 31/2 Jahre dauernde eruptive Erregung des Mont Pelé zerfällt in der Hauptsache in einen ersten, durch die mehr oder weniger verheerenden Ausbrüche (8. und 20. Mai, 30. Aagust, ferner am 26. Mai, 6. Juni und 9. Juli) des Sommers 1902 bezeichneten Abschnitt und in eine zweite Periode, während deren sich die Tätigkeit vorzugsweise auf die Bildung der Staukuppe und der Felsnadel unter zeitweisen, aber immer schwächeren Ausbrüchen heisser Wolken beschränkte. Bald nachdem sie ihre höchste Erhebung erreicht hatte, ist die berühmte Fels. nadel in sich zusammengestürzt, die Bildung der Staukuppe aber danerte noch an und auch die Hebungen an der Stelle der seit August 1903 verschwundenen Nadel setzten sich weiterhin fort und bewirkten vorzugsweise eine Ausdehnung des Domes und die Bildung kleinerer Nadeln an der Stelle der verschwundenen. Der Druck der von unten nachdrängenden Massen und die Zusammenziehung bei der Erstarrung gaben den Anlass zur Entstehung zahlloser. Risse und zu einer allmählichen und vollständigen Zerbröckelung der vordem von Spitzen und Zacken gekrönten Andesitmasse, die bald nichts anderes mehr sein wird als ein grosser Blockhaufen, dessen Schutt allmählich auch die Ausfüllung der Rinne zwischen dem Dom und der ihn umschliessenden Kraterwand bewirken wird. Der Boden dieser Rinne lag zuletzt noch etwa $60 \mathrm{~m}$ unter der Umrandung des Kraterkessels; der Gipfel des Domes überragt jetzt die letztere noch um $120 \mathrm{~m}$ und besitzt eine absolute Höhe von $1355 \mathrm{~m}$ gegenüber einer.höchsten Erhebung der ehemaligen Nadel von $1620 \mathrm{~m}$ am 31. Mai 1908. Damals ragte die letztere $550 \mathrm{~m}$ hoch über den Boden der Caldera und $385 \mathrm{~m}$ üluer den alten Gipfel des Berges empor. Der grösste Durchmesser des Kraterkessels beträgt $1050 \mathrm{~m}$.

Bezüglich der den Dom zusammensetzenden Andesite ergab sich, dass sich die einer raschen Abküblung entsprechenden glasreichen Gesteine, welche ehedem die Oberfläche des Domes gebildet haben dürften, jetzt nur mehr an dessen Basis finden, während in den höheren Teilen die mehr kristallinen, teilweise quarzfïhrenden Gesteinstypen herrschen, die glasigen dagegen völlig fehlen. Es wird daraus geschlossen, dass die dem jetzigen Gipfel benachbarten Teile des Domes nicht mehr durch Lavaerguss oder Intrusion, sondern entsprechend der Bildung der ehemaligen grossen Felsnadel, durch Extrusion fester, bereits in einiger Tiefe erstarrten Massen gebildet wurden. LACRoIX trägt im Anschlusse hieran eine Theorie über die Entstehung des Quarzes in diesen Gesteinen vor, die dahin geht, dass sich dieses Mineral, entsprechend seiner begrenzten Existenzfähigkeit bis zur Temperatur von etwa $800^{\circ}$, erst in dem schon stark abgekühlten Magma unter dem Einfluss von Wasserdämpfen auf das zähe Magma gebildet habe, während der Tridymit bei höheren Temperataren aus dem Gesteinsglase wabrscheinlich infolge einer Art Entglasung und möglicherweise gleichzeitig ebenfalls durch Hydropneumatolyse entstanden sei. Im übrigen weist Verf. auf die grosse petrographische Verwandtschaft des Mont Pelé-Gesteins mit 
den Dioritporphyriten so zahlreicher amerikanischer und europäischer Lakkolithe und Batholithe hin. Sie wird dereinst noch augenscheinlicher werden, wenn. die Denudation, die in etwas grösserer Tiefe erstarten Intrusionen an den Tag gebracht haben wird.

Seit der Eruption von Santorin im Jahre 1866 war die Bildung einer echten Staukuppe durch Nachschab zäbflüssiger Lava nicht mebr beobachtet worden. Sie ist jedenfalls ein so seltenes Phänomen, dass Lacrolx aus seiner eigenen Erfahrung nur wenige analoge Beispiele anzuführen vermag, nämlich die Pitons du Carbet und einige andere dazitische Berge auf Martinique, die dem "Dom" des Mont Pelé so ausserordentlich ähnliche Soufrière von Guadeloupe und vielleicht auch den Puy de Dôme und einige andere "Puys" der Auvergne.

Der Abschnitt über die heissen Wolken der Mont Pelé-Eruptionen und ein Vergleich derselben mit den Schntt- und Aschenlawinen der Vesuveruption 1906 gibt dem Verf. Veranlassung, auch auf die verscbiedenen, schon seit längerer Zeit unterschiedenen Typen vulkanischer Eruptionen einzugehen. Über die "nuées ardentes" des Mont Pelé hatte sich LACrorx schon sehr ansfuhrlich in seinem eingangs erwähnten Hauptwerke geäussert. Er hält auch jetzt noch daran fest, dass die verheerenden heissen Wolken, deren erster St. Pierre mitsamt seinen fast 30000 Einwohnern zum Opfer gefallen ist, zumeist aus einer jeweils sich öffnenden Spalte in dem Andesitdom seitlich und mit seitlicher Richtung hervorbrachen und dass ihre lebendige Kraft in erster Linie der Explosion selbst, in zweiter der durch die Schwerkraft bedingten lawinenartigen Bewegung $\mathrm{za}$ zuschreiben war. Im Gegensatz zu anderen Eruptionen, gab es am Mont Pelé keinen eigentlichen ringsum geschlossenen Krater, die Explosionen erfolgten nicht nach oben, sondern nach der Seite und zwar fast immer in der Richtung eines tiefen Taleinschnittes, der Rivière blanche, durch den die fortgeschleuderten Massen mehrere Kilometer weit längs einer nur um wenige Grade geneigten Schussbahn und im ganzen unabhängig vom Bodenrelief dahinstürmten. Diese vulkanischen Wolken unterschieden sich wesentlich von den Lawinen und Schuttströmen, wie sie von ANDERSor und FLETT gelegentlich der Eruption der Soufrière auf St. Vincent im Jahre 1902 beschrieben worden sind. Hier wie am Vesuv, wo sich am Abhange des Kegels zeitweise die locker und steil liegenden Auswurfsmassen loslösten, als Schuttlawinen nach abwärts stürzten und dabei tiefe Furchen auf der Oberfläche des Berges erzeugten, handelte es sich um vulkanisches Material, das zuerst emporgeschleudert und dann auf dem Rand des Kraters niedergefallen war.

Nach der Heftigkeit und Häufigkeit der Auswürfe nnd nach der Form der Auswurfsprodukte unterscheidet man die vulkanischen Explosionen in eine Anzahl von Typen; ihre Sonderheiten sind lediglich bedingt durch den Grad der Leichteilissigkeit des boteiligten Magmas. Im ganzen mag ein gewisser Typus für einen bestimmten Vulkan charakteristisch sein, aber schon der Vesav lebrt, dass zu verschiedenen Zeiten verschiedene Explosionstypen am gleichen Vulkan, je nach dem augenblicklichen Flüssigkeitsgrad seiner Lava, in Erscheinung treten können. Indem er das bereits von anderen gegebene Schema etwas erweitert, unterscheidet Lacrorx folgeade Explosionstypen:

1. Ausserordentlich leichtflüssige basaltische Laven werden in Form von Fontänen, seltener unter Bildung von Auswürflingen und äusserst selten unter heftiger Explosion. emporgeschleudert. Die Auswürflinge sind sehr blasig; die 
äusserst leichtfüssige Lava wird beim Emporspritzen sogar in Fäden ausgezogen („Peles Haar").

Typus Hawaï (nach Friedländer and Agdilar).

2. Etwas grössere Zähflüssigkeit führt zum Auswurf von blasigen Schlacken unter heftigen Explosionen. Im Krater ist die Oberfläche der Lava noch nicht erstarrt. Einschlüsse festen, älteren Gesteins geben der sie umhüllenden Lava die Form der "Bombe".

Typus Stromboli (nach Mercalti).

3. Das an sich schwerflüssige Magma ist im Krater oberflächlich schon so weit zähe geworden, dass die hervorbrechenden Gasmassen von Zeit zu Zeit eine widerstandsfähige Oberfächenschicht zerschmettern müssen. Die schweren Explosionen erzeugen die dichten, in wirbelnden Ballen sich ausdehnenden Wolken, deren Gestalt man mit einem Blumenkohl verglichen hat. Die Auswurfsprodukte sind eckige, innen oft aufgeblähte und äusserlich gesprungene Blöcke mit manchmal gewaltigen Dimensionen (Brotkrustenbomben) und massenhafter feiner Staub. Je grösser die Last festen Materials ist, welches diese Wolken emporreissen, um so häufger sind in ibnen elektrische, durch Reibung erzeugte Entladungen.

Typus Vulcano (nach Mercalli).

4. Der vierte Typus hat wie der Typus Valcano eine grosse Zähigkeit des Magmas zur Voraussetzung, unterscheidet sich aber von ihm durch die heissen, nach abwärts stürmenden Wolken. LACRoIX unterscheidet hier zwei Fälle:

a) "Die Explosion ist nach oben gerichtet, die heissen Auswürflinge, die mit Gasen und Dämpfen gemischt die Wolke bilden, steigen zuerst senkrecht empor und fallen dann, im Gegensatz zu den vulkanianischen Wolken, die sich zunächst ihrer schwersten Bestandteile entledigen and dann in der Luft zerteilen, in ihrer ganzen Masse mit Wucht auf die Flanken des Berges nieder und rollen mit zunehmender Geschwindigkeit nach Art einer Lawine auf seinen Abhängen nieder." Nach Lacrorx beruht die Beweglichkeit dieser Lawine darauf, dass sie gewissermassen eine „Emulsion gasförmiger und fester Stoffe" darstellen soll. Solcher Art waren die heissen Wolken der Soufrière vou St. Vincent und einige wenige des Mont Pelé.

b) „Die Explosion ist nach der Seite gerichtet. Die Wolke ist der vorigen zwar ähnlich; statt aber vertikal aufzusteigen und dann vermöge ibrer Schwere niederzustürzen, nimmt sie von Anfang an einen von oben nach unten gerichteten Weg, entsprechend der Lage der Ausbrachsöffnung (die jeweils an der Seite des Doms entsteht, vergl. oben); zu der Wucht des Ausbruches addiert sich die Schwerkraft, weil sie in demselben Sinne wirkt. Diese richtunggebende Kraft der Explosion hatte in den verschiedenen beobachteten Wolken eine sehr wechselnde Bedeutang, manchmal war sie fast null."

Diesen Typus bezeichnet LackoIx mit einem schon von Anderson und FLETT gebrauchten Ausdruck als den peléanischen (Type peléen).

Man sieht, dass sich im grossen ganzen die explosiven Ausserungen der Vulkane auf zwei Haupttypen zurückführen lassen, nämlich auf solche, bei denen leichtflüssige, d. h. vorzugsweise basaltische, und in solche, bei welchen zähe Magmen, d. h. vor allem andesitische, dazitische und liparitische, im Spiele sind. Diese beiden Typen wären dann der strombolianische und der vulkanianische. Aber auch zwischen diesen ist eine scharfe Scheidung nicht möglich, und 
am gleichen Vulkane sind, wie oben gesagt, gelegentlich beide Typen und Übergänge zwischen beiden ( möglich.

Es sei hier.vergleichsweise die Unterscheidung der Typen angeführt, wie sie Mercalti (zuletzt in seinem Buche I vulcani attivi della terra, Mailand 1907) vorgenommen hat:

1. 'Typus Stromboli oder Hawaï, gleich Lacnotx' 1 und 2.

2. Gemischter Typus, steht zwischen Lacrorx' 2 und 3.

3. Typus Vulcano, gleich Lacrorx' 3.

4. Ultravalkanianischer Typus. Damit sind solche Explosionen gemeint, die überhaupt nur älter es, zerschmettertes Gestein entführen und keine jüngeren Laven fördern, also ledighich durch die plötzliche Ausdehnung von hochgradig gespannten Gasmassen entstehen. Solcher Art soll die furchtbare Eruption des Bandaï San in Japan gewesen sein.

Zum Schlusse geht Verf. noch ausführlicher auf die Frage ein, ob etwa Pompei in derselben Weise wie Saint Pierre vernichtet worden sei. Letztere Stadt fand ihren Untergang unter dem Ansturm einer heissen Wolke des „type peléen", sie warde niedergeblasen, die Einwohner erstickten; die Katastrophe spielte sich in wenig Augenblicken ab. In derselben Weise sollte nach dem amerikanischen Geographen HEILPRIN auch Pompei zerstört worden sein. Zahlreiche Tatsachen, die schon einem einigermassen aufmerksamen Besucher der italienischen Ruinenstadt nicht, entgehen können, und dazu anch Peinios' sehr ausführlicher und anschaulicher Bericht beweisen, dass diese Annahme nicht zutrifft. Pompei ist verschïttet worden, und die Katastrophe des Jahres $79 \mathrm{n}$. Chr. hat sich nur durch ihre Heftigkeit, nicht aber nach ihrer Art von dem letzten Vesuvausbruch des Jabres 1906 unterschieden.

Auch diese schöne Veröffentlichung LAcrorx' ist wiederum mit zahlreichen Abbildungen ausgestattet, die den Anblick so vieler, gerade in den Tropen mehr oder weniger vergänglicher vulkanischer Gebilde für immer festhalten und überliefern werden.

\title{
Das Deckgebirge des produktiven Karbons in Westfalen, am Niederrhein und in Holland nach neueren Untersuchungen.
}

\author{
Von N. Tilmann (Bonn). \\ Mit einer Karte im Text.
}

\section{Literatur.}

Die Entwickelung des niederrheinisch-westfälischen Steinkohlenbergbaus in der zweiten Hälfte des 19. Jahrhunderts. I. Geologie von Cremer und Mentzer. Berlin 1903. (Enthält ein Literaturverzeichnis bis 1903.)

Mentzel, H.: Übersicht über die neuere Literatur über das niederrheinisch-west-

fälische Kohlenbecken. Glückauf. 43. Jahrg. Nr. 36/37, S. 1216. (Literatur 1903-1907.)

$$
1908 .
$$

1. Ahururg: Die Ergebnisse der neveren Tiefbohrungen im östlichen Holland. Glückauf. 44. Jahrg. S. 1205. 